\title{
A Novel HIV-1 Nef Mutation in a Primary Pediatric Isolate Impairs MHC-Class I Downregulation and Cytopathicity
}

\author{
Ayub Ali, ${ }^{1,2}$ Robert L. Furler, ${ }^{3}$ Livia Pedroza-Martins, ${ }^{4}$ Arnaud D. Colantonio, ${ }^{1,5}$ Deborah Anisman-Posner, ${ }^{1,5}$ \\ Yvonne Bryson, ${ }^{1,6}$ Otto O. Yang, ${ }^{1,2}$ and Christel H. Uittenbogaart ${ }^{1,5-7}$
}

\begin{abstract}
HIV-1-induced cytopathicity of thymocytes is a major cause of reduced peripheral $\mathrm{T}$ cells and rapid disease progression observed in HIV-1-infected infants. Understanding the virulence factors responsible for thymocyte depletion has paramount importance in addressing the pathogenesis of disease progression in children. In this study, thymocyte depletion was analyzed following infection with two primary CXCR4-tropic HIV-1 pediatric isolates (PI), PI-2 and PI-2.1, which were serially derived from an in utero-infected infant. Although highly similar to each other, PI-2 showed markedly decreased thymocyte depletion in vitro compared with PI-2.1. Further analysis showed a novel deletion in the Nef protein (Nef $\Delta$ K7S) of PI-2, which was absent in PI-2.1. This deletion inhibited Nef-mediated major histocompatibility complex class I (MHC-I) downregulation in infected thymocytes in vitro and in vivo; in contrast, the mutated Nef continued to downregulate CD4 surface expression in vitro. These results suggest that HIV-1 Nef contributes to thymic damage in infants through selective functions.
\end{abstract}

Keywords: HIV-1, Nef, MHC-I downregulation, cytopathicity, thymocyte

\section{Introduction}

$\mathbf{T}$ HE THYMUS IS PARTICULARLY ACTIVE during early life as the immune system develops in infants. Thymocyte infection with HIV-1 leads to severe thymic involution and dysfunction, resulting in declined thymopoiesis and subsequent depletion of peripheral T lymphocytes. ${ }^{1}$ Although direct killing of thymocytes has been suggested as a major mechanism responsible for thymic dysfunction, ${ }^{2,3}$ HIV-1 also causes nonspecific activation and proliferation of intrathymic progenitor cells through secretion of proinflammatory cytokines in the thymic microenvironment that result in elevated viral infection, exhaustion of precursor cells, and destruction of thymic architecture. ${ }^{4,5}$ These conditions within the thymus have been observed in children infected with HIV-1 and are suggested to be a leading cause of rapid disease progression in perinatally infected children. ${ }^{5,6}$ Studies to understand the underlying pathological and molecular aspects of thymus dysfunction in HIV-1 infection suggest that the Nef protein of
HIV-1 plays a key role in impairing thymocyte development in addition to interfering with peripheral T cell function. ${ }^{7-10}$

Nef is a phosphorylated and myristoylated $27 \mathrm{kDa}$ protein that is unique to HIV/SIV and is relatively conserved with a $75 \%-85 \%$ similarity within clade B of HIV $-1 .{ }^{11}$ Nef is a multifunctional protein that is suggested to play multiple key roles in HIV-1 and SIV pathogenesis. ${ }^{8,12}$ Deletion of Nef markedly attenuates SIV pathogenicity in the rhesus macaque model of AIDS, ${ }^{8,13}$ and dysfunctional Nef has been associated with delayed disease progression in humans. ${ }^{14-16} \mathrm{Nef}$ has also been shown to impair T cell development ${ }^{10,17}$ and enhance viral replication and infectivity. ${ }^{18,19}$ Its expression begins early in the viral life cycle and localizes to the plasma membrane, cytoplasm, and within the budding virions. Nef promotes viral infectivity, immune evasion, and replication capacity by altering the activation state of infected cells. It does so by modulating cell signal transduction pathways and altering cell surface expression of receptors. Nef regulates various cell signaling molecules, such as Hck, Lck, Fyn, Lyn,

${ }^{1}$ UCLA AIDS Institute, University of California, Los Angeles, Los Angeles, California.

${ }^{2}$ Division of Infectious Diseases, Department of Medicine, University of California, Los Angeles, Los Angeles, California.

${ }^{3}$ Division of Infectious Diseases, Department of Medicine, Weill Cornell Medicine, New York, New York.

${ }^{4}$ ANRS, French National Agency for Research on AIDS and Viral Hepatitis, Paris, France.

${ }^{5}$ Department of Microbiology, Immunology \& Molecular Genetics, University of California, Los Angeles, Los Angeles, California.

${ }^{6}$ Department of Pediatrics, University of California, Los Angeles, Los Angeles, California.

${ }^{7}$ Jonsson Comprehensive Cancer Center, University of California, Los Angeles, Los Angeles, California. 
Vav, Src, PAK1/2 (NAK), MAPK (Erk1), c-Raf1 kinase, $\mathrm{PI} 3 \mathrm{~K}$, and Ask $1 .{ }^{20,21}$ Nef downregulates cell surface receptors such as $\mathrm{CD} 4,{ }^{22-24}$ major histocompatibility complex class I (MHC-I), ${ }^{23-25}$ MHC-II, and MHCII-associated invariant chain (Ii, CD74), ${ }^{26} \mathrm{CD} 28,{ }^{27}$ and host restriction factor SERINC5. ${ }^{28}$

Nef-mediated downregulation of surface expression of MHC-I and CD4 has been extensively studied in vitro. The downregulation of MHC-I A and B molecules protects infected cells from CTL-mediated killing. ${ }^{29-31}$ Previously, we described two closely related CXCR4-tropic HIV-1 primary isolates [pediatric isolates (PI)-2.1 and PI-2] serially derived from an in utero-infected infant, and their dissimilar abilities to cause thymocyte depletion. ${ }^{32} \mathrm{PI}-2.1$ showed aggressive thymocyte depletion in vitro, while isolate PI-2 showed diminished cytopathicity. Given the central role of Nef in HIV-1 disease progression, we performed a detailed analysis of Nef sequences of these two pediatric isolates and their corresponding abilities to downregulate MHC-I and CD4.

\section{Materials and Methods}

\section{HIV-1 pediatric isolates and virus stocks}

HIV-1 primary isolates and plasma samples for virus isolation were obtained as close as possible to birth from 10 of 34 children born to 204 HIV-1-seropositive mothers monitored prospectively in the Los Angeles Pediatric AIDS Consortium between June 1988 and May $1995 .^{32-34}$ The isolates were characterized as syncytium inducing (SI) or nonsyncytium inducing (NSI) by using the MT-2 assay. ${ }^{35}$ The characteristics of some of the children providing the viral isolates were previously described: PI-2 is an SI HIV-1 isolate obtained 1 week after birth from an infant infected in utero who developed AIDS and died within 6 months. ${ }^{34}$ PI-2.1 was isolated from plasma 1 month after birth from the same infant. PI-2 and PI-2.1 isolation and propagation were done by directly adding serum from an infected infant to a pool of purified activated allogeneic $\mathrm{CD} 4^{+} \mathrm{T}$ cells, prepared as described previously. ${ }^{36,37}$ Briefly, allogeneic $\mathrm{CD}^{+}$ $\mathrm{T}$ cells from three normal donors were individually purified by capture in CD4 monoclonal antibody-coated tissue culture flasks (Applied Immune Sciences, Santa Clara, CA) and activated by stimulation with $200 \mathrm{ng} / \mathrm{mL}$ anti-human CD3 antibody (OKT3; Ortho Biotech, Inc., Raritan, NJ) plus $5,000 \mathrm{U} / \mathrm{mL}$ recombinant human interleukin-2 (IL-2) for 5 days. Cells from three donors were combined, cryopreserved in liquid nitrogen, and then thawed and cultured in medium with IL-2 for 2-3 days before infection. The makeup by phenotype of the $\mathrm{CD} 4^{+} \mathrm{T}$ cell pool at the time of infection by the pediatric isolates was as follows: $95 \% \mathrm{CD}^{+} \mathrm{CD}^{-}, 5 \%$ $\mathrm{CD}^{+} \mathrm{CD}^{+},<1 \% \mathrm{CD}^{+} \mathrm{CD}^{-}, 60 \% \mathrm{CD} 27^{+}$, and $50 \%$ HLA$\mathrm{DR}^{+}$. In addition, $94 \%$ of the cells from the stimulated $\mathrm{CD} 4^{+}$ $\mathrm{T}$ cell pool expressed CXCR4 and 30\%-40\% expressed CCR5 (data not shown). ${ }^{32}$ The same $\mathrm{CD}^{+} \mathrm{T}$ cell pool was used for isolating PI-2 and PI-2.1 virus from plasma (passage 1) and for preparing virus stocks (passage 2) and for preparing the control viruses including CCR5-tropic virus HIV-1 NFNSX (NF-NSX) and the CXCR4-tropic virus HIV-1 NL4-3 (NL4-3). ${ }^{38,39}$ The infectivity of the pediatric isolates and of the molecular-clone-derived laboratory viruses was determined by limiting dilution assays in PBMC. The CCR5-tropic pediatric isolate PI- 8 was prepared similarly to PI-2 and PI2.1 and used as control for the Western blot studies. ${ }^{32}$

\section{Cloning and sequencing of the nef gene in pediatric HIV-1 isolates}

RNA was isolated from plasma using the TRIzol reagent (Invitrogen, Carlsbad, CA) and was reverse transcribed into complementary DNA (cDNA) using the High-Capacity cDNA Reverse Transcription Kits (Applied Biosystems, Foster City, CA). Reverse transcription was primed with a random primer (Applied Biosystems) and following nested polymerase chain reaction (PCR), the cDNA of full-length nef was amplified. First and second rounds of PCR were carried out using Nef 8687F (5-GTAGCTGAAGGGACAGATAGGGTTAT-3) and Nef 9589R (5-TAGTTAGCCAGAGAGCTCCCA-3) as the outer primer set and Nef 8787 XbalF (5-GCTCTAGAATG GGTGGCAAGTGCTCAA-3) and Nef 9495R (5-TTATATG CAGCATCTGAGGGC-3) as the inner primer set, respectively. Numbering of primers is based on the NL4-3 sequence. High Fidelity Advantage ${ }^{\circledR}$ HD Polymerase Mix (Clontech, Mountain View, CA) was used for PCR, according to the manufacturer's instructions. After cloning of PCR products into the Topo TA cloning vector (Invitrogen), plasmid DNAs were isolated from at least six colonies for sequencing with the ABI 3130 Genetic Analyzer (Applied Biosystems).

\section{Production of single-round infectious, pseudotyped HIV-1 reporter virus}

The reporter HIV-1 construct AA1305\#18 plasmid containing an NL4-3 backbone with reporter antigens heat shock antigen (HSA) or mouse CD24 (mCD24) and deletions in Vpu, Env, and Vpr was used to produce VSV-G pseudotyped virus as described previously. ${ }^{40}$ Insertion of isolated nef genes was done using PCR products that were gel purified with Quick Spin Column (Qiagen, Valencia, CA) and digested with $X b a \mathrm{I}$ and $B s p$ EI restriction enzymes (New England Biolabs, Ipswich, MA). Single-round infectious pseudovirus was produced by cotransfecting $293 \mathrm{~T}$ cells with the whole-genome plasmid AA1305\#18 containing nef alleles and a plasmid encoding the envelope glycoprotein from VSV-G using BioT transfection reagent (Bioland, Paramount, CA).

\section{Western blot}

HIV-1 nef genes from the pediatric isolates (PI) were cloned into a plasmid containing the NL4-3 genome (pNL43 ) to make infectious virus as described previously. ${ }^{41}$ To construct NL4-3/PI-nef, the nef gene of NL4-3 was replaced with either individual nef obtained from Topo cloning or quasispecies or nef obtained from the nested PCR as described above. T1 cells were infected with NL4-3 virus containing nef alleles from either wild-type NL4-3 or alleles from the pediatric isolates (multiplicity of infection [MOI] = 1). On day 5, lysates harvested from the infected cells were run onto 10\% polyacrylamide gel electrophoresis (PAGE) for protein separation. Following transfer to a PVDF membrane (Millipore, Billerica, MA), HIV-1 proteins were detected by an anti-Nef antibody (Nef\#2949; NIH AIDS Reagent Program) or an anti-Gag antibody (Gag\#6457; NIH AIDS Reagent Program) followed by detection using the SuperSignal 
West Pico Chemiluminescent Substrate (Thermo Scientific, Rockford, IL).

\section{Immunophenotyping}

Surface expression of MHC-I and CD4 in HIV-1-infected and uninfected cells was done using flow cytometry. Study of MHC-I downregulation by Nef on infected thymocytes ${ }^{40,42}$ with FITC- and PE-conjugated monoclonal antibodies was done as previously described. ${ }^{42-45}$ PE-conjugated or FITCconjugated KC57 mAb (Beckman Coulter, Brea, CA) was used to determine intracellular $\mathrm{HIV}-1_{\mathrm{Gag}}$ protein expression in distinct thymocyte subsets ${ }^{43,46}$ and was performed following surface immunophenotyping. ${ }^{44,45}$ Study of CD4 downregulation by Nef on infected cells was described previously. ${ }^{40}$ Briefly, $2 \times 10^{6}$ T1 cells were infected with $100 \mu \mathrm{L}$ (10 ng p24) of viral stock. Three days postinfection, surface expression of $\mathrm{CD} 4$ and $\mathrm{mCD} 24$ was detected with $\mathrm{PE}-$ conjugated human anti-CD4 (BioLegend, San Diego, CA) and FITC-conjugated mouse anti-CD24 (BioLegend). Multiparameter data acquisition was done on a dual-laser FACS Calibur flow cytometer (BDIS, San Jose, CA) or an MACSQuant VYB, and data analysis was performed with FCS Express 6.0.

\section{In vitro infection of postnatal thymocytes}

Normal human postnatal thymus specimens were obtained from infants and children undergoing corrective cardiac surgery. The tissue was cut into small pieces, red blood cells were removed by $\mathrm{NH}_{4} \mathrm{Cl} /$ Tris lysis buffer, and the mechanically disrupted tissue was passed over a cell strainer to generate a single-cell suspension of thymocytes as previously described. $^{39,44,46}$ Thymocytes were infected in vitro with CXCR4-tropic pediatric isolates (PI-2 and PI-2.1), the CXCR4-tropic molecular clone NL4-3, the CCR5-tropic molecular clone NF-NSX, or mock infected with the supernatant from the same $\mathrm{CD} 4^{+} \mathrm{T}$ cell pool used for virus stock preparation. The thymocytes were cultured in serum-free medium for 14 days with IL-2 and IL-4 as previously described. ${ }^{32}$ All thymocyte infections with pediatric isolates were standardized by using equal numbers of virus infectious units $/ 10^{7}$ cells. HIV-1 virion production was measured by HIV-1 Gag p24 enzyme-linked immunosorbent assay as previously described. ${ }^{44}$

\section{In vivo infection of SCID-hu mice}

C.B-17 SCID mice were bred at UCLA and implanted with human fetal thymus and liver graft (thy/liv) under the murine kidney capsule. ${ }^{47}$ Four to six months postimplantation, the thy/liv grafts of SCID-hu mice implanted with thy/liv grafts from the same donor were infected with different HIV-1 isolates (PI-2, PI-2.1, NL4-3, or NFN-SX) by injecting 10$30 \mathrm{ng}$ of p24 in a 50- $\mu \mathrm{L}$ volume directly into the graft as previously described. ${ }^{44}$ Mock-infected implants, used as controls in the experiments, were prepared by injecting implants with $50 \mu \mathrm{L}$ of appropriate control supernatants. Two different experiments consisting of 10-12 SCID-hu mice per 3 -week time point were conducted. MHC-I and CD4 phenotyping in HIV-1-infected cells was done at 3 weeks postinfection as previously described. ${ }^{45}$

\section{Results}

$\mathrm{Pl}-2$ is less cytopathic to thymocytes in vitro than PI-2.1 or NL4-3

HIV-1 strains isolated from in utero and perinatally infected children vary in rates of disease progression and exhibit differential effects on thymopoiesis during in vitro culture. ${ }^{6,32}$ Beyond the observation that CXCR4-tropic HIV isolates cause more thymocyte depletion than CCR5-tropic HIV isolates ${ }^{48}$ it is unclear what molecular mechanisms determine differential effects on thymocyte depletion and thymic development by HIV-1. Two CXCR4-tropic HIV-1 isolates (PI-2 and PI-2.1) were isolated from an in uteroinfected infant and were found to infect $\mathrm{CD} 4^{+}$thymocytes differently. ${ }^{32}$ Primary human thymocytes were infected in vitro with PI-2, PI-2.1, or NL4-3 viruses in parallel with a mock-infection control and cultured in serum-free medium supplemented with IL-2 and IL-4 for 14 days. Flow cytometric analysis of surface CD4 and CD8 expression was done on the thymocytes to measure subset depletion. Apoptotic and dead cells were excluded using 7-AAD, and quadrants were set using isotype controls. Following in vitro infection of human thymocytes, PI-2 was less efficient in depleting $\mathrm{CD}^{+}$thymocytes (9\% $\mathrm{CD} 4^{+}$depletion) compared with PI$2.1\left(40 \% \mathrm{CD}^{+}\right.$depletion) or the CXCR4-tropic molecular clone NL4-3 (90\% $\mathrm{CD}^{+}$depletion) as related to the percentage of $\mathrm{CD}^{+}$cells in mock-infected thymocytes (Fig. 1A). Despite initial infection with equal infectious units, PI-2 in vitro replication was also lower than PI-2.1 as measured by p24 levels in the culture supernatants (Fig. 1B).

\section{Pl-2 contains amino acid mutations in the Nef protein}

The Nef protein was previously shown to affect the pathogenicity of HIV-1 in cultured thymocytes ${ }^{17}$ and the thy/liv implant in the SCID-hu mouse model. ${ }^{49}$ Sequencing of the $n e f$ alleles found in PI-2 and PI-2.1 was done to examine a possible cause of their discrepancy in thymic cytopathicity. Analysis of the two nef sequences revealed that the amino acids (aa) of these two isolates were highly similar, and the majority of reported functional motifs were conserved in both isolates (Fig. 2A). The myristoylation residue (G2) required for membrane localization, the oligomerization domain needed for all functions, and motifs such as $\mathrm{WL}_{58}, \mathrm{LL}_{165}, \mathrm{DD}_{175}$, and $\mathrm{EE}_{155}$, which are required for CD4 binding and lysosomal degradation function, were found to be preserved in both isolates. However, a deletion of seven amino acids (Nef $\Delta$ K7S) from amino acid positions 82 to $88\left({ }_{82} \mathrm{KGALDLS}_{88}\right)$ was present in PI-2 but not in PI-2.1. This sequence falls within the core domain of Nef (aa 72-203), which is a relatively conserved and structured type II polyproline helix (aa 72-77) followed by two $\alpha$-helices (aa 81-120). ${ }^{50}$ This novel deletion is also 4 amino acids downstream of a polyproline helix $\left({ }_{72} \mathrm{PXXPXR} / \mathrm{K}_{77}\right)$ binding site for Srchomology domain 3 (SH3) that mediates cellular activation and MHC-I downregulation. ${ }^{15,29}$ In addition to Nef $\Delta \mathrm{K} 7 \mathrm{~S}$, a single amino acid mutation at position 71 from K to R (K71R), adjacent to the SH3 binding site, was observed in PI-2.

\section{Nef mutations in Pl-2 do not inhibit expression of structural proteins}

To assess if the identified mutations in the nef gene of PI-2 inhibited Nef protein expression, Western blot analysis was 
A MOCK: $77 \%$ CD4+

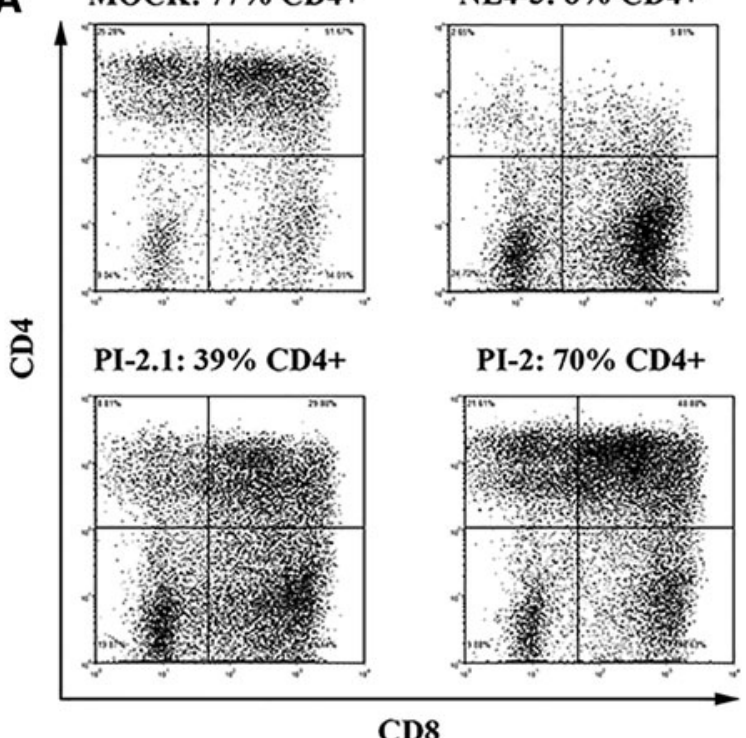

CD8

\section{B PI-2 Shows Reduced Infection of Human Thymocytes}

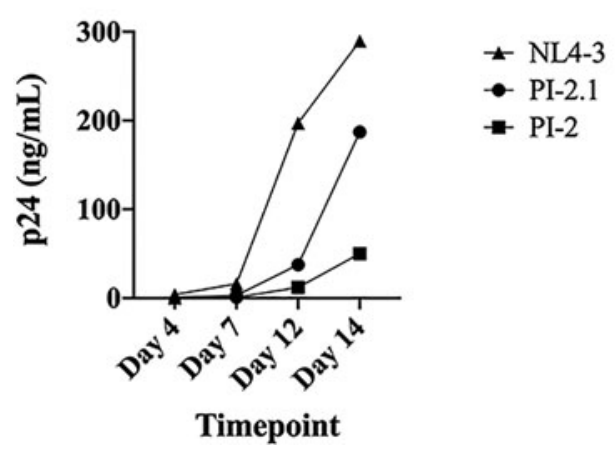

FIG. 1. Thymocytes infected with the CXCR4-tropic HIV isolate PI-2 in vitro show less CD4 depletion and lower HIV-Gag p24 levels than thymocytes infected with PI-2.1 or NL4-3. Thymocytes were infected in vitro with CXCR4tropic pediatric isolates (PI-2 and PI-2.1), the CXCR4-tropic NL4-3 molecular clone NL4-3, or mock infected with the supernatant from the same cells $\left(\mathrm{CD}^{+} \mathrm{T}\right.$ cell pool). (A) Fourteen days postinfection, thymocytes were stained for CD4 and CD8. Apoptotic and dead cells were excluded using 7-AAD, and quadrants were set using isotype controls. (B) Viral replication was followed by measuring HIV Gag p24 antigen in the supernatants. PI, pediatric isolates.

done on cells infected with CXCR4-tropic molecular clone NL4-3 (lane 1), CCR5-tropic pediatric isolate PI- $8^{32}$ (lane 2), and CXCR4-tropic isolate PI-2 (lane 3). The results show that PI-2-infected cells expressed similar levels of HIV-1 Gag (Fig. 2B) and Nef (Fig. 2C) as cells infected with PI-8 or NL4-3 and used as controls. Accordingly, PI-2-infected cells express HIV-1 Nef despite mutations.

\section{Pl-2 is impaired in MHC-I downregulation in vitro and in vivo}

While a myriad of cellular effects have been shown for Nef, two of its most clearly defined functions are to downregulate cell surface expression of MHC-I and CD4 mole- cules. These functions were assessed in thymocyte cultures infected with PI-2 and PI-2.1 and compared with cultures infected with the HIV-1 molecular clones NL4-3 or NFNSX (NL4-3 with a substituted CCR5-tropic Env from strain JR-FL). Mock-infected cultures were prepared by adding supernatants of uninfected activated allogeneic $\mathrm{CD} 4^{+} \mathrm{T}$ cell pools. Thymocytes infected in vitro (Fig. 3) were analyzed for surface expression of MHC-I. Following infection, MHC-I downregulation was assessed by the mean fluorescence intensity (MFI) of MHC-I in HIV-1 $\mathrm{Gag}^{+}$cells $\left(\mathrm{KC} 7^{+}\right)$. The cells were surface stained with antibodies to MHC-I (HLA-ABC-FITC) and CD3-APC followed with intracellular staining for HIV-1 Gag proteins using the KC57-PE antibody. Isotype controls were used to set the quadrants. MHC-I expression on mock-infected thymocytes is shown in Figure 3A. Cells productively infected $\left(\mathrm{KC}^{+} 7^{+}\right)$with CXCR4tropic NL4-3 containing a functional Nef (Fig. 3B) had decreased expression of MHC-I $(\mathrm{MFI}=263)$ compared with uninfected $\left(\mathrm{KC}^{-} 7^{-}\right)$cells $(\mathrm{MFI}=745)$. Cells infected with the CCR5-tropic virus NF-NSX containing a functional Nef (Fig. 3C) had a decreased expression of MHC-I $(\mathrm{MFI}=217)$ compared with uninfected cells $(\mathrm{MFI}=551)$. Cells infected with the pediatric isolate PI-2.1 containing a functional Nef (Fig. 3D) also had decreased MHC-I expression $(\mathrm{MFI}=220)$ compared with uninfected cells $(\mathrm{MFI}=639)$. However, cells infected with the pediatric isolate PI-2 containing the Nef mutation (Fig. 3E) showed a smaller decrease in MHC-I surface expression $(\mathrm{MFI}=417)$ compared with uninfected cells $(\mathrm{MFI}=573)$.

The ability of the pediatric isolate Nef proteins to downregulate MHC-I was also tested in vivo using an SCID-hu mouse model implanted with human fetal thy/liv tissue that forms a thymus-like organoid as previously described. ${ }^{44,45}$ SCID-hu mice were infected with NL4-3, PI-2, PI-2.1, or mock-negative control supernatant. MHC-I downregulation in productively infected $\left(\mathrm{KC} 57^{+}\right)$thymocytes was measured ex vivo at 3 weeks postinfection by measuring the HLA-ABC MFI. As shown in Figure 4A, cells infected with NL4-3 ( $n=5$ mice) and PI-2.1 ( $n=5$ mice) showed decreased mean surface expression of HLA-ABC compared with cells infected with PI-2 ( $n=6$ mice). PI-2 expressing thymocytes had similar levels of MHC-I expression as mock infection $(n=5$ mice). Statistical analysis was done using two-tailed unpaired $\mathrm{t}$ tests. Similar to the in vitro experiments, infections of thy/liv implants with PI-2, PI-2.1, or NL4-3 again demonstrated diminished capabilities of PI-2 to downregulate MHC-I following infection, whereas PI-2.1 exhibited comparable MHC-I downregulation with NL4-3 (Fig. 4A). The percentages of $\mathrm{CD}^{+}$expression on thymocytes in thy/liv implants following infection with PI-2 were the same as in mock-infected implants, contrary to our earlier in vitro experiments. However, PI-2.1-infected implants showed a similar decrease in the percentage of $\mathrm{CD}^{+}$thymocytes as NL4-3-infected implants (Fig. 4B).

\section{HIV-1 PI-2 Nef downregulates CD4 but not MHC-I expression in the absence of Vpu and Env in vitro}

To investigate the small discrepancy in CD4 downregulation between our in vitro and in vivo experiments, we analyzed the Nef proteins separately from other HIV-1 proteins, Env and Vpu, which have previously been reported 
PI-2 MGNIWSKRSLGGWPKVRERMRQAEPAAEGVGEVSRDLEKHGA ITSS--TANSTACTWLEA PI-2 . 1 MGNIWSKRSLGGWPKVRERMRQAEPAAEGVGEVSRDLEKHGA I TSS--TANSTACTWLEA NL4-3 MGGKWSKSSVI GWPAVRERMRRAEPAADGVGAVSRDLEKHGA I TSSNTAANNAACAWLEA

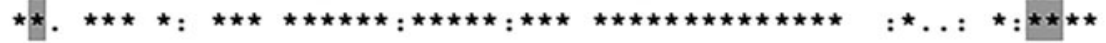

PACS Binding

$$
\begin{array}{ll}
\text { SH3 } & \text { Binding } \\
\hline 70 \quad 80
\end{array}
$$

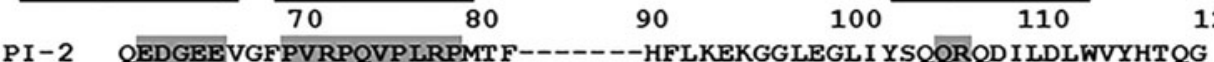

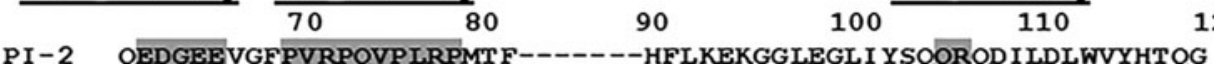

$\begin{array}{lllcl}\frac{70}{70} & 80 & 90 & 100 & 110\end{array}$ PAK Binding PI - 2.1 QEDGEEVGFPVKPQVPLRPMT FKGALDLSHFLKEKGGLEGLI YSQQRQD ILDLWVYHTQG NL4-3 QEE-EEVGFPVTPQVPLRPMT YKAAVDLSHFLKEKGGLEGL I HSQRRQD ILDLWI YHTQG

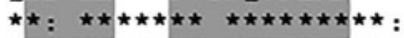

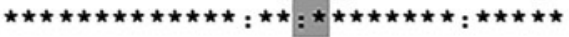

\section{oligomerization} 130 $140 \frac{\text { COP-I Binding }}{150}$

$\underline{\text { AP1/2 Binding }} \mathrm{V1H}$ Binding

PI-2 YFPDWQNYTQG PGVRY PLCFGWCFKLVPVEKEKVEEA TERENTSLLH PINLHGMEDPEKE PI - 2.1 YFPDWQNYTQG PGVRY PLCFGWCFKLV PVEKE KVEEATERENTSLLH PINLHGMED PEKE NL4-3 YFPDWQNYTPGPGVRY PLTFGWCYKLVPVEPDKVEEANKGENTSLIHPVSLHGMDDPERE

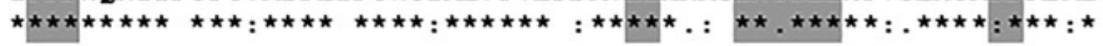

190 200

PI-2 VLMWKFDSRLAFHHMARELHPEYFKNPI - 2.1 VLMWKFDSRLAFHHMARELHPEYFKNNL4-3 VLEWRFDSRLAFHHVARELHPEYFKNC

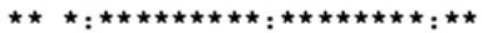
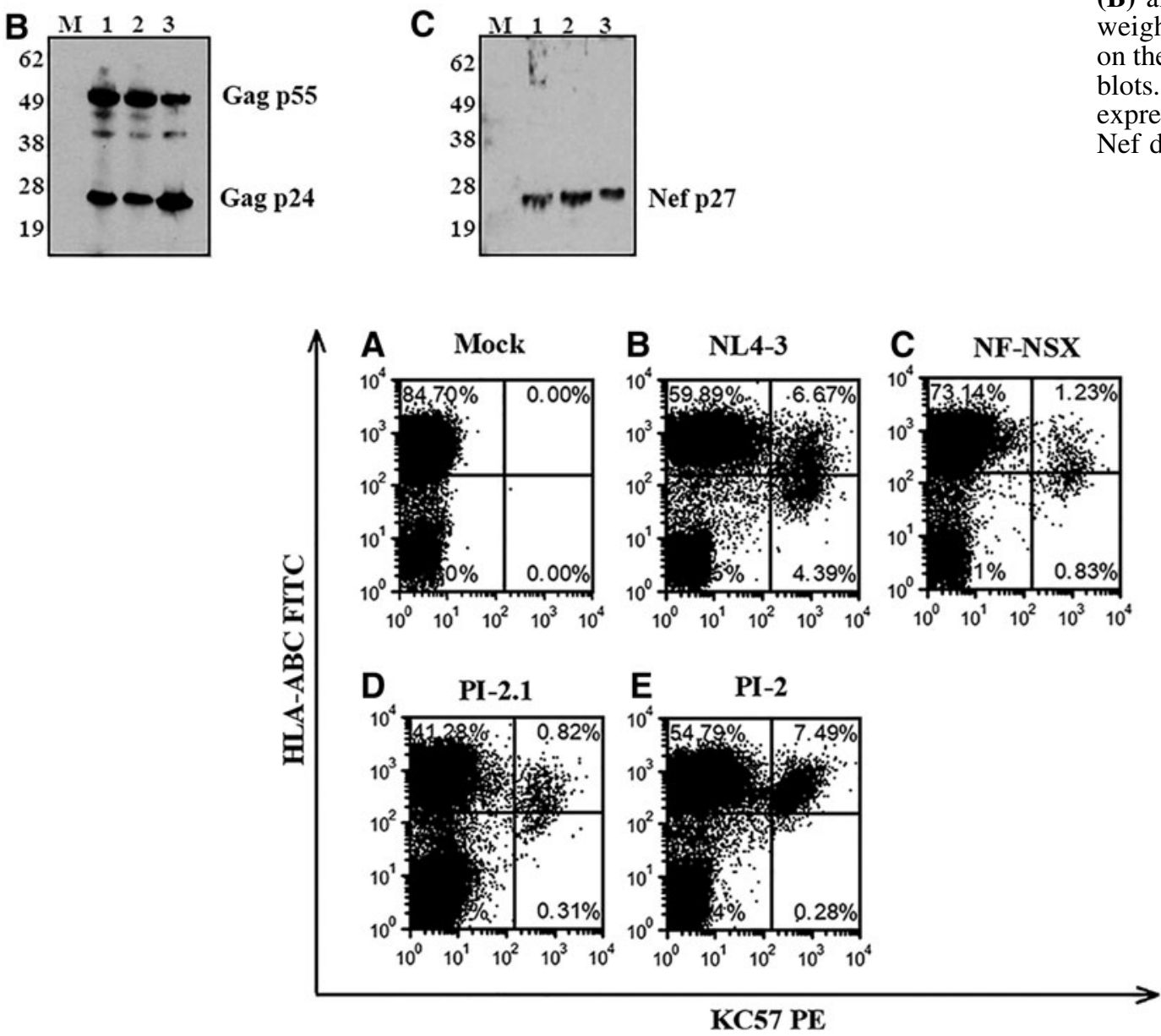

FIG. 3. Pediatric HIV isolate 2 (PI-2) is impaired in MHC-I downregulation in vitro. Thymocytes were infected in vitro with CXCR4-tropic pediatric isolates (PI-2 and PI-2.1), the CXCR4-tropic NL4-3 and CCR5-tropic NF-NSX molecularly cloned HIV-1 isolates, or mock infected with the supernatant from the same cells $\left(\mathrm{CD} 4^{+} \mathrm{T}\right.$ cell pool). (A-E) MHC-I downregulation was measured to assess Nef function by using the geometric MFI of MHC-I (HLA-ABC) in KC57 ${ }^{+}$(HIV-1 $\mathrm{Gag}^{+}$) cells. (A) Mock infected negative control; (B) CXCR4-tropic virus NL4-3; (C) CCR5-tropic virus NF-NSX; (D) pediatric isolate PI-2.1; and (E) pediatric isolate PI-2. MFI, mean fluorescence intensity; MHC-I, major histocompatibility complex class I. 
A

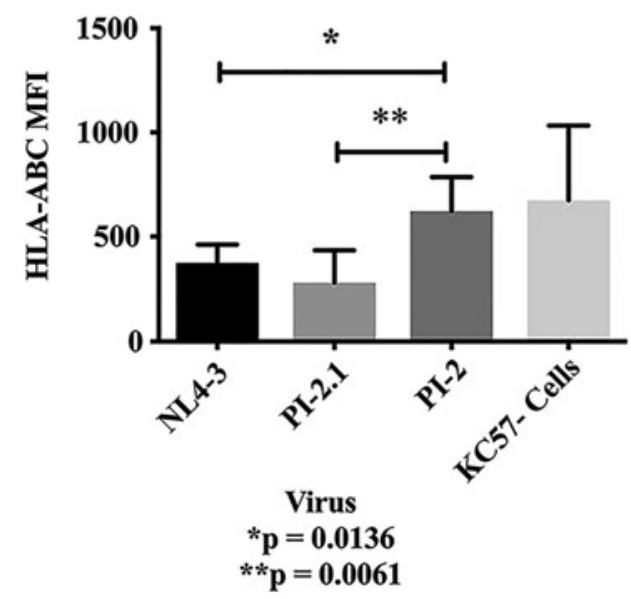

B

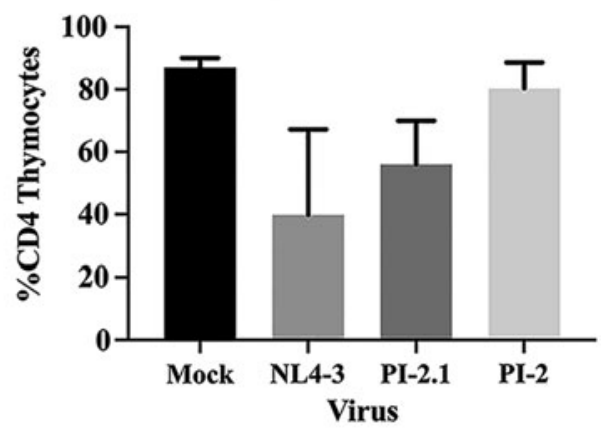

FIG. 4. Pediatric HIV isolate 2 (PI-2) is impaired in MHC-I downregulation in vivo, but is less cytopathic than PI-2.1. SCID mice implanted with human fetal thy/liv (SCID-hu mice) were infected with NL4-3 or pediatric isolates PI-2.1 and PI2. (A) MHC-I downregulation was measured ex vivo at 3 weeks postinfection. The MFI values show the levels of MHC-I on productively infected $\left(\mathrm{KC}_{5} 7^{+}\right)$thymocytes. Cells infected with NL4-3 ( $n=5$ mice), PI-2.1 ( $n=5$ mice), and PI-2 $(n=6$ mice). Error bars depict standard error of the mean. Statistical analysis was done using two-tailed unpaired $t$ tests. PI-2 expressing thymocytes had similar levels of MHC-I expression as mock infection ( $n=5$ mice, data not shown). (B) Percentages of thymocytes expressing CD4 were measured ex vivo at 3 weeks postinfection. The percentages of CD4 ${ }^{+}$ thymocytes in PI-2 infected are similar to the mock-infected thy/liv implants. thy/liv, thymus/liver.

to contribute to CD4 surface downregulation. ${ }^{51,52}$ To test the effect of Nef separately from Env and Vpu while maintaining their physiologic expression levels, PI-2 and PI-2.1 nef genes were inserted into an NL4-3-based genome containing deletions of env and $v p u .^{40}$ Single-cycle infection was done using VSV-G pseudotyped viruses expressing a $\mathrm{CD} 24$ tag and the various nef genes. In agree- ment with our earlier in vitro data obtained in thymocytes infected with the original pediatric isolates, PI-2 Nef was unable to downregulate MHC-I in infected cells as much as Nef from PI-2.1 and NL4-3 (Fig. 5A-E). Confirming our previous experiment, PI-2 Nef was still able to downregulate CD4 surface expression in vitro, even in the absence of Vpu and Env (Fig. 5F-J).
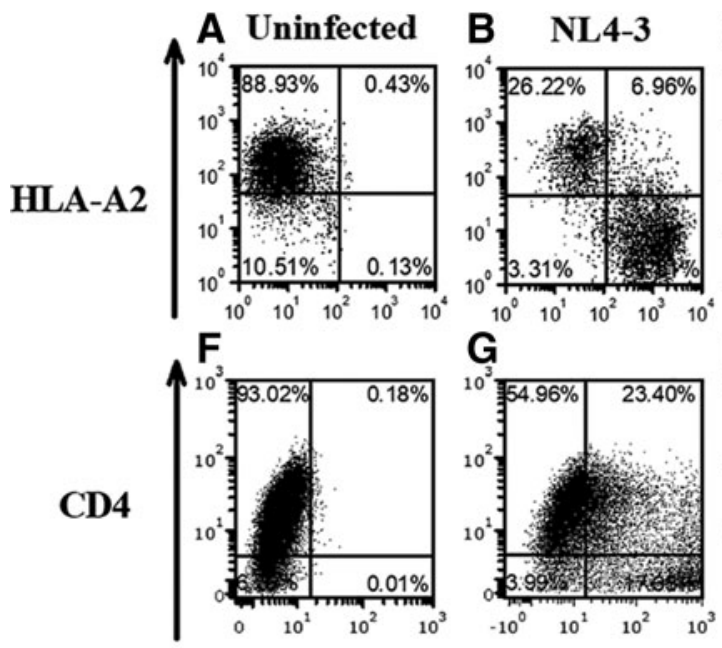

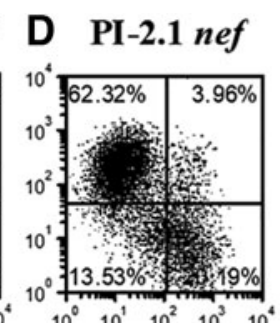

$\mathrm{H}$
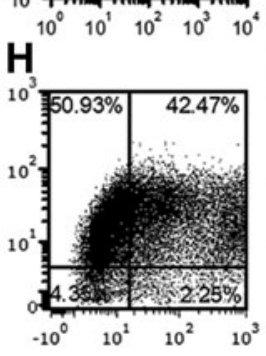

I

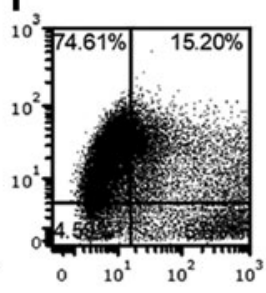

E PI-2 nef

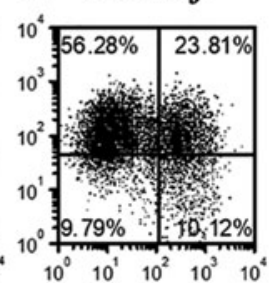

J

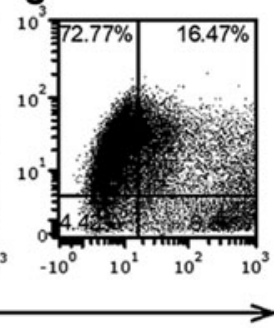

CD24

FIG. 5. HIV PI-2 Nef downregulates CD4 but not MHC-I expression in the absence of Vpu, Env, and Vpr. To assess the MHC-class I downregulation of the nef genes in pediatric isolates PI-2.1 and PI-2 in the absence of Vpu, Env, and Vpr proteins, the nef genes were cloned out of the PI-2.1, PI-2, and NL4-3 isolates and placed in an NL4-3 vector lacking Vpu, Env, and Vpr. To determine the effect of PI-2.1 and PI-2 Nef proteins on MHC-I and CD4 downregulation, VSV-G pseudotyped virus, including a CD24 tag and the various nef genes, was used to infect T1 cells. HLA-A2 and CD4 surface expression in T1 cells infected with (A, F) mock control; (B, G) VSV-G Nef pseudotyped defective NL4-3 lacking Vpu, Env, and Vpr; (C, H) NL4-3 $\Delta$ Nef; (D, I) VSV-G pseudotyped PI-2.1 nef lacking vpu, env, and vpr; or (E, J) VSV-G pseudotyped PI-2 nef lacking vpu, env, and vpr. 


\section{Discussion}

Following comparison of two primary HIV-1 pediatric isolates from the same in utero-infected infant, PI-2 and PI-2.1, we identified a unique in-frame deletion Nef $\Delta$ K7S in PI-2, which attenuates the depletion of thymocytes in vitro. Nef proteins bearing this mutation are defective for MHC-I downregulation, but this effect is not due to reduced Nef expression or global Nef dysfunction. Nef proteins bearing this mutation were still able to downregulate CD4 surface expression in vitro. This novel $\Delta \mathrm{K} 7 \mathrm{~S}$ in-frame deletion is in a location that is not known to contain any specific motifs required for previously defined Nef functions. However, it is adjacent to the $\mathrm{SH} 3$ binding site that is critical for multiple Nef functions, including cellular activation and MHC-I downregulation. ${ }^{53,54}$ It is possible that $\Delta \mathrm{K} 7 \mathrm{~S}$ alters the tertiary structure of Nef and thus interferes with interactions with host factors. While there is a $\mathrm{K}$ versus $\mathrm{R}$ polymorphism that falls within the PXXP motif between residues $72-75$ when comparing PI-2.1 with PI-2, this is unlikely to contribute to impaired MHC-I downregulation by PI-2. Although the NL4-3 Nef sequence has T71 (Fig. 2A), the subtype B consensus sequence is actually $71 \mathrm{R}$, and thus, PI-2 actually matches "wildtype" Nef more closely than PI-2.1 or NL4-3 at position 71 . Moreover, K71R is a conservative substitution. Although highly unlikely, however, we cannot definitively rule out that $71 \mathrm{R}$ somehow contributed to the impaired MHC-I downregulation by PI-2.

Previous data show that various mutations in Nef affect oligomerization as well as ternary complex formation with cell molecules such as AP-1 and MHC-I, ${ }^{50,55-57}$ and a survey of in vivo Nef sequences demonstrated frequent examples of Nef with impaired MHC-I downregulation despite no identifiable mutations in the defined motifs involved in this function. ${ }^{58}$ Similarly, the significance of the K71R substitution mutation in PI-2 Nef is unclear. This mutation is also located in proximity to the canonical sequence required for SH3 binding. Mutational studies have demonstrated that residues flanking the motif play critical role in determining binding specificity to $\mathrm{SH} 3$ domain. ${ }^{59}$

Others have reported that Nef-mediated CD4 downregulation correlates with increased viral pathogenesis in thymocytes, and MHC-I downregulation has diminished influence in cytopathicity. ${ }^{60}$ In contrast, our findings indicate that PI-2-mutated nef containing virus was less cytotoxic in thymocytes than PI-2.1, despite having a similar ability to downregulate surface expression of CD4 in vitro. PI-2infected cells did not downregulate MHC-I but did decrease CD4 surface expression in vitro, indicating that the novel identified mutations in PI-2 Nef may not have affected binding to several host factors reported to be involved in CD4 downregulation. ${ }^{61,62}$ Point mutations in the nef gene (WL57AA, LL164AA, and DD174AA) of the NL4-3 molecular clone abrogate CD4 downregulation and decrease Nef-mediated pathogenesis. ${ }^{60}$ These mutations were not present in PI-2. Van Nuffel et al. have previously reported that Nef:PAK2 and an intact VGF motif within Nef are required for reduced thymic output. ${ }^{63}$ Despite having intact VGF and PAK2 binding motifs and representative CD4 downregulation in vitro, PI-2 still lacked the virulence and thymic depletion seen in thymocytes infected with PI-2.1 or molecular clones, indicating a correlation between MHC-I downregulation and Nef's ability to deplete thymocytes.
Given the importance of MHC-I molecules in T cell development, it is unclear whether the defect in MHC-I downregulation by HIV isolate PI-2 is directly related to the attenuated thymic cytopathicity, or if the novel identified mutations disrupt another pathway that potentiates the negative effect of Nef in the thymopoiesis. Further unraveling this phenomenon may provide greater insight into the particularly rapid disease progression seen in HIV-1-infected children, and may offer therapeutic targets for this particularly vulnerable population.

\section{Sequence Data}

The nucleotide sequences for PI-2 and PI-2.1 nef genes have been deposited in GenBank under accession numbers KC702883 and KC702882, respectively.

\section{Acknowledgments}

The following reagents were obtained from the NIH AIDS Reagent Program, Division of AIDS, NIAID, NIH: anti-Nef antibody (Nef\#2949), anti-Gag antibody (Gag\#6457), and the HIV-1 NL4-3 infectious molecular clone (pNL4-3) from Dr. Malcolm Martin (cat. no. 114). ${ }^{38}$ The content is solely the responsibility of the authors and does not necessarily represent the official views of the NIH.

\section{Authors' Contributions}

A.A., L.P.-M., R.L.F., O.O.Y., and C.H.U. conceived and designed the experiments; A.A., L.P.-M., A.D.C., and D.A.-P. performed the experiments; A.A., L.P.-M., R.L.F., A.D.C., D.A.-P., and C.H.U. analyzed the data; Y.B. and O.O.Y. contributed reagents/materials/analysis tools and advice; A.A., R.L.F., O.O.Y., and C.H.U. wrote the article.

\section{Author Disclosure Statement}

The authors do not have any financial or nonfinancial competing interests.

\section{Funding Information}

This work was supported by the National Institutes of Health, grant numbers: HD29341, AI054286, AI32440, and CA016042. This work was also supported by the UCLA AIDS Institute, UCLA Center for AIDS Research (CFAR) NIH AI28697, James B. Pendleton Charitable Trust, and the McCarthy Family Foundation.

\section{References}

1. Douek DC, Betts MR, Hill BJ, et al.: Evidence for increased T cell turnover and decreased thymic output in HIV infection. J Immunol 2001;167:6663-6668.

2. Bonyhadi ML, Rabin L, Salimi S, et al.: HIV induces thymus depletion in vivo. Nature 1993;363:728-732.

3. Hanna Z, Kay DG, Rebai N, Guimond A, Jothy S, Jolicoeur P: Nef harbors a major determinant of pathogenicity for an AIDS-like disease induced by HIV-1 in transgenic mice. Cell 1998;95:163-175.

4. Bandera A, Ferrario G, Saresella M, et al.: $\mathrm{CD}^{+} \mathrm{T}$ cell depletion, immune activation and increased production of regulatory $\mathrm{T}$ cells in the thymus of HIV-infected individuals. PLoS One 2010;5:e10788. 
5. Su L, Kaneshima H, Bonyhadi M, et al:: HIV-1-induced thymocyte depletion is associated with indirect cytopathogenicity and infection of progenitor cells in vivo. Immunity 1995;2:25-36.

6. Kourtis AP, Ibegbu C, Nahmias AJ, et al.: Early progression of disease in HIV-infected infants with thymus dysfunction. N Engl J Med 1996;335:1431-1436.

7. Pennington DJ, Jenkins SA, Brady HJ, Miles CG, Dzierzak EA, Abraham DJ: HIV-I Nef severely impairs thymocyte development and peripheral T-cell function by a CD4independent mechanism. Genes Funct 1997;1:321-335.

8. Kestler HW, 3rd, Ringler DJ, Mori K, et al.: Importance of the nef gene for maintenance of high virus loads and for development of AIDS. Cell 1991;65:651-662.

9. Skowronski J, Parks D, Mariani R: Altered T cell activation and development in transgenic mice expressing the HIV-1 nef gene. EMBO J 1993;12:703-713.

10. Stove V, Naessens E, Stove C, Swigut T, Plum J, Verhasselt B: Signaling but not trafficking function of HIV-1 protein Nef is essential for Nef-induced defects in human intrathymic T-cell development. Blood 2003;102:29252932.

11. Jubier-Maurin V, Saragosti S, Perret JL, et al.: Genetic characterization of the nef gene from human immunodeficiency virus type 1 group $M$ strains representing genetic subtypes A, B, C, E, F, G, and H. AIDS Res Hum Retroviruses 1999;15:23-32.

12. Arien KK, Verhasselt B: HIV Nef: Role in pathogenesis and viral fitness. Curr HIV Res 2008;6:200-208.

13. Daniel MD, Kirchhoff F, Czajak SC, Sehgal PK, Desrosiers RC: Protective effects of a live attenuated SIV vaccine with a deletion in the nef gene. Science 1992;258:1938-1941.

14. Kirchhoff F, Greenough TC, Brettler DB, Sullivan JL, Desrosiers RC: Brief report: Absence of intact nef sequences in a long-term survivor with nonprogressive HIV-1 infection. N Engl J Med 1995;332:228-232.

15. Deacon NJ, Tsykin A, Solomon A, et al.: Genomic structure of an attenuated quasi species of HIV-1 from a blood transfusion donor and recipients. Science 1995;270:988991.

16. Learmont JC, Geczy AF, Mills J, et al: Immunologic and virologic status after 14 to 18 years of infection with an attenuated strain of HIV-1. A report from the Sydney Blood Bank Cohort. N Engl J Med 1999;340:1715-1722.

17. Verhasselt B, Naessens E, Verhofstede C, et al.: Human immunodeficiency virus nef gene expression affects generation and function of human $\mathrm{T}$ cells, but not dendritic cells. Blood 1999;94:2809-2818.

18. Harris M: HIV: A new role for Nef in the spread of HIV. Curr Biol 1999;9:R459-R461.

19. Zuo J, Suen J, Wong A, et al.: Functional analysis of HIV type 1 Nef gene variants from adolescent and adult survivors of perinatal infection. AIDS Res Hum Retroviruses 2012;28:486-492.

20. Landi A, Iannucci V, Nuffel AV, Meuwissen P, Verhasselt B: One protein to rule them all: Modulation of cell surface receptors and molecules by HIV Nef. Curr HIV Res 2011; 9:496-504.

21. Geyer M, Fackler OT, Peterlin BM: Structure-Function relationships in HIV-1 Nef. EMBO Rep 2001;2:580-585.

22. Garcia JV, Miller AD: Serine phosphorylation-independent downregulation of cell-surface CD4 by nef. Nature 1991; 350:508-511.
23. Mann JK, Byakwaga H, Kuang XT, et al.: Ability of HIV-1 Nef to downregulate CD4 and HLA class I differs among viral subtypes. Retrovirology 2013;10:100.

24. Mann JK, Chopera D, Omarjee S, et al.: Nef-mediated down-regulation of CD4 and HLA class I in HIV-1 subtype $\mathrm{C}$ infection: Association with disease progression and influence of immune pressure. Virology 2014;468-470:214225.

25. Schwartz O, Marechal V, Le Gall S, Lemonnier F, Heard JM: Endocytosis of major histocompatibility complex class I molecules is induced by the HIV-1 Nef protein. Nat Med 1996;2:338-342.

26. Stumptner-Cuvelette P, Morchoisne S, Dugast M, et al.: HIV-1 Nef impairs MHC class II antigen presentation and surface expression. Proc Natl Acad Sci U S A 2001;98: 12144-12149.

27. Swigut T, Shohdy N, Skowronski J: Mechanism for downregulation of CD28 by Nef. EMBO J 2001;20:1593-1604.

28. Rosa A, Chande A, Ziglio S, et al: HIV-1 Nef promotes infection by excluding SERINC5 from virion incorporation. Nature 2015;526:212-217.

29. Collins KL, Chen BK, Kalams SA, Walker BD, Baltimore D: HIV-1 Nef protein protects infected primary cells against killing by cytotoxic T lymphocytes. Nature 1998; 391:397-401.

30. Ali A, Ng HL, Dagarag MD, Yang OO: Evasion of cytotoxic $\mathrm{T}$ lymphocytes is a functional constraint maintaining HIV-1 Nef expression. Eur J Immunol 2005;35:3221-3228.

31. Mwimanzi F, Toyoda M, Mahiti M, et al:: Resistance of major histocompatibility complex class B (MHC-B) to Nef-mediated downregulation relative to that of MHC-A is conserved among primate lentiviruses and influences antiviral T cell responses in HIV-1-infected individuals. J Virol 2018;92:1-19.

32. Pedroza-Martins L, Boscardin WJ, Anisman-Posner DJ, Schols D, Bryson YJ, Uittenbogaart CH: Impact of cytokines on replication in the thymus of primary humAan immunodeficiency virus type 1 isolates from infants. J Virol 2002;76:6929-6943.

33. Ganeshan S, Dickover RE, Korber BT, Bryson YJ, Wolinsky SM: Human immunodeficiency virus type 1 genetic evolution in children with different rates of development of disease. J Virol 1997;71:663-677.

34. von Seidlein L, Plaeger S, Dickover R, Krogstad P, Stiehm ER, Bryson Y: Primary human immunodeficiency virus type 1 infection during pregnancy associated with transmission of SI/MT-2 cell tropic virus and precipitous loss of CD4 cells in mother and infant. Pediatr Infect Dis J 1998; 17:528-530.

35. Koot M, Vos AH, Keet RP, et al: HIV-1 biological phenotype in long-term infected individuals evaluated with an MT-2 cocultivation assay. AIDS 1992;6:49-54.

36. Ferbas J, Kaplan AH, Hausner MA, et al.: Virus burden in long-term survivors of human immunodeficiency virus (HIV) infection is a determinant of anti-HIV $\mathrm{CD}^{+}$lymphocyte activity. J Infect Dis 1995;172:329-339.

37. Dickover RE, Dillon M, Leung KM, et al.: Early prognostic indicators in primary perinatal human immunodeficiency virus type 1 infection: Importance of viral RNA and the timing of transmission on long-term outcome. J Infect Dis 1998;178:375-387.

38. Adachi A, Gendelman HE, Koenig S, et al.: Production of acquired immunodeficiency syndrome-associated retrovirus 
in human and nonhuman cells transfected with an infectious molecular clone. J Virol 1986;59:284-291.

39. Pedroza-Martins L, Gurney KB, Torbett BE, Uittenbogaart $\mathrm{CH}$ : Differential tropism and replication kinetics of human immunodeficiency virus type 1 isolates in thymocytes: Coreceptor expression allows viral entry, but productive infection of distinct subsets is determined at the postentry level. J Virol 1998;72:9441-9452.

40. Ali A, Realegeno S, Yang OO, Lewis MJ: Simultaneous assessment of CD4 and MHC-I downregulation by Nef primary isolates in the context of infection. J Virol Methods 2009;161:297-304.

41. Ali A, Jamieson BD, Yang OO: Half-genome human immunodeficiency virus type 1 constructs for rapid production of reporter viruses. J Virol Methods 2003;110:137-142.

42. Schmid I, Uittenbogaart CH, Keld B, Giorgi JV: A rapid method for measuring apoptosis and dual-color immunofluorescence by single laser flow cytometry. J Immunol Methods 1994;170:145-157.

43. Schmid I, Uittenbogaart $\mathrm{CH}$, Giorgi JV: A gentle fixation and permeabilization method for combined cell surface and intracellular staining with improved precision in DNA quantification. Cytometry 1991;12:279-285.

44. Gurney KB, Colantonio AD, Blom B, Spits H, Uittenbogaart $\mathrm{CH}$ : Endogenous IFN-alpha production by plasmacytoid dendritic cells exerts an antiviral effect on thymic HIV-1 infection. J Immunol 2004;173:7269-7276.

45. Gurney KB, Uittenbogaart $\mathrm{CH}$ : Human immunodeficiency virus persistence and production in T-cell development. Clin Vaccine Immunol 2006;13:1237-1245.

46. Uittenbogaart $\mathrm{CH}$, Anisman DJ, Jamieson BD, et al.: Differential tropism of HIV-1 isolates for distinct thymocyte subsets in vitro. AIDS 1996;10:F9-F16.

47. Aldrovandi GM, Feuer G, Gao L, et al:: The SCID-hu mouse as a model for HIV-1 infection. Nature 1993;363: $732-736$.

48. Correa R, Munoz-Fernandez MA: Viral phenotype affects the thymic production of new $\mathrm{T}$ cells in HIV-1-infected children. AIDS 2001;15:1959-1963.

49. Jamieson BD, Aldrovandi GM, Planelles V, et al:: Requirement of human immunodeficiency virus type 1 nef for in vivo replication and pathogenicity. J Virol 1994;68: 3478-3485.

50. Arold S, Hoh F, Domergue S, et al:: Characterization and molecular basis of the oligomeric structure of HIV-1 nef protein. Protein Sci 2000;9:1137-1148.

51. Kerkau T, Bacik I, Bennink JR, et al.: The human immunodeficiency virus type 1 (HIV-1) Vpu protein interferes with an early step in the biosynthesis of major histocompatibility complex (MHC) class I molecules. J Exp Med 1997;185:1295-1305.

52. Yewdell JW, Hill AB: Viral interference with antigen presentation. Nat Immunol 2002;3:1019-1025.

53. Madrid R, Janvier K, Hitchin D, et al.: Nef-induced alteration of the early/recycling endosomal compartment cor- relates with enhancement of HIV-1 infectivity. J Biol Chem 2005;280:5032-5044.

54. Coleman SH, Madrid R, Van Damme N, et al.: Modulation of cellular protein trafficking by human immunodeficiency virus type 1 Nef: Role of the acidic residue in the ExxxLL motif. J Virol 2006;80:1837-1849.

55. Noviello CM, Benichou S, Guatelli JC: Cooperative binding of the class I major histocompatibility complex cytoplasmic domain and human immunodeficiency virus type 1 Nef to the endosomal AP-1 complex via its mu subunit. J Virol 2008;82:1249-1258.

56. Wonderlich ER, Williams M, Collins KL: The tyrosine binding pocket in the adaptor protein 1 (AP-1) mu1 subunit is necessary for Nef to recruit AP-1 to the major histocompatibility complex class I cytoplasmic tail. J Biol Chem 2008;283:3011-3022.

57. Singh RK, Lau D, Noviello CM, Ghosh P, Guatelli JC: An MHC-I cytoplasmic domain/HIV-1 Nef fusion protein binds directly to the mu subunit of the AP-1 endosomal coat complex. PLoS One 2009;4:e8364.

58. Lewis MJ, Balamurugan A, Ohno A, Kilpatrick S, Ng HL, Yang OO: Functional adaptation of Nef to the immune milieu of HIV-1 infection in vivo. J Immunol 2008;180: 4075-4081.

59. Kuo LS, Baugh LL, Denial SJ, et al.: Overlapping effector interfaces define the multiple functions of the HIV-1 Nef polyproline helix. Retrovirology 2012;9:47.

60. Stoddart CA, Geleziunas R, Ferrell S, et al.: Human immunodeficiency virus type 1 Nef-mediated downregulation of CD4 correlates with Nef enhancement of viral pathogenesis. J Virol 2003;77:2124-2133.

61. Landi A, Timermans CG, Naessens E, Vanderstraeten H, Stove V, Verhasselt B: The human immunodeficiency virus (HIV) Rev-binding protein (HRB) is a co-factor for HIV-1 Nef-mediated CD4 downregulation. J Gen Virol 2016;97: 778-785.

62. Landi A, Vermeire J, Iannucci V, et al:: Genome-wide shRNA screening identifies host factors involved in early endocytic events for HIV-1-induced CD4 down-regulation. Retrovirology 2014;11:118.

63. Van Nuffel A, Arien KK, Stove V, et al.: Primate lentiviral Nef proteins deregulate T-cell development by multiple mechanisms. Retrovirology 2013;10:137.

Address correspondence to: Christel H. Uittenbogaart Department of Microbiology, Immunology \& Molecular Genetics University of California, Los Angeles 615 Charles E. Young Drive South Los Angeles, CA 90095

E-mail: uittenbo@ucla.edu 